\title{
Un passage de relai en toute sérénité
}

Depuis plus de trente ans, notre rédacteur en chef Christian Libersa, a toujours terminé en temps et en heure le bouclage des numéros de Thérapie.

Mu par la passion de faire partager notre discipline, il a conçu avec soin la maquette de chaque numéro autour d'un thème d'actualité. Ainsi nous avons bénéficié de mises au point régulières et didactiques sur des sujets aussi divers que les hypolipémiants, les immunosuppresseurs, la réglementation des essais cliniques... Ces articles ont été et sont des références pour notre enseignement. Qui n'a pas attendu avec fébrilité la publication des comptes rendus de Giens ?

En lui accordant une place d'honneur, il a permis à la pharmacovigilance française d'avoir un organe d'expression. Au demeurant, la publication relative à la méthode d'imputabilité en pharmacovigilance contribue à l'impact factor.

Certes, il y a eu des années difficiles, Christian Libersa a maintenu le cap. Trouvant un nouvel éditeur, il a offert à Thérapie une renaissance sous la couverture bleue que nous lui connaissons actuellement.

Thérapie jouit d'un rayonnement international à l'heure où la langue de Shakespeare envahit la littérature scientifique. Quel plaisir de recevoir des demandes d'abonnements des pays francophiles et francophones mais aussi des pays du Soleil-Levant, démontrant combien le travail de Christian Libersa et des personnes qui l'ont suivi dans cette fantastique aventure, a été de qualité.

Comment a-t-il pu tenir la distance ? Je suppose que les courses à vélo sur l'enfer des pavés du Nord lui ont forgé son endurance, sa ténacité et sa volonté de porter haut la revue Thérapie.

Homme modeste, d'une grande discrétion, Christian n'aime pas les hommages. Cependant, je tiens, au nom des membres du conseil d'administration et de tous les lecteurs, à le remercier très sincèrement pour son engagement plein et entier pour que vive Thérapie.

De façon plus personnelle, je souhaite souligner le soutien fidèle qu'il m'a témoigné depuis mes premières années en pharmacologie.

Christian Libersa a remis à Jean-Louis Montastruc les clefs de Thérapie avec élégance, à l'image de l'homme qu'il est. Nul doute que notre nouveau rédacteur en chef conservera l'esprit de notre revue et son éthique.

Tous les pharmacologues et les thérapeutes se joignent à moi pour te dire, cher Christian : MERCI !

Professeur Marie-Christine Perault-Pochat

Présidente de la Société Française de Pharmacologie et de Thérapeutique SFPT 